\title{
Simple Method of Forest Type Inventory by Joining Low Resolution Remote Sensing of Vegetation Indices with Spatial Information from the Corine Land Cover Database
}

\author{
Jarosław J. Zawadzki, ${ }^{1}$ Karol Przeździecki, ${ }^{1}$ Karol Szymankiewicz, ${ }^{1}$ \\ and Wojciech Marczewski ${ }^{2}$ \\ ${ }^{1}$ Department of Environmental Engineering, Warsaw University of Technology, Ulica Nowowiejska 20, 00-653 Warsaw, Poland \\ ${ }^{2}$ Space Research Centre, Polish Academy of Sciences, Ulica Bartycka 18A, 00-716 Warsaw, Poland \\ Correspondence should be addressed to Jarosław J. Zawadzki; j.j.zawadzki@gmail.com
}

Received 20 December 2012; Accepted 26 January 2013

Academic Editors: N. Frascaria-Lacoste, G. Martinez Pastur, and J. F. Negron

Copyright (C) 2013 Jarosław J. Zawadzki et al. This is an open access article distributed under the Creative Commons Attribution License, which permits unrestricted use, distribution, and reproduction in any medium, provided the original work is properly cited.

\begin{abstract}
The paper presents a simple, inexpensive, and effective method allowing for frequent classification of the forest type coniferous, deciduous, and mixed using medium and low resolution remote sensing images. The proposed method is based on the set of vegetation indices such as NDVI, LAI, FAPAR, and LAIxCab calculated from MODIS and MERIS satellite data. The method uses seasonal changes of the above-mentioned vegetation indices within annual cycle. The main idea was to collect and carefully analyse seasonal changes in vegetation indices in a given ecosystem type proven by a Corine Land Cover, 2006 database, and to compare them afterwards with those of a particular forest under study. Each type of a forest ecosystem has its own specific dynamics of development, thus enabling recognition of the type by comparing temporal changes of the proposed measures based on vegetation indices. Temporal measures of changes were created for selected reference stands by the ratios of particular indices determined in July and April, which are the middle and the beginning of a vegetation season in Poland, respectively. The analysed vegetation indices were additionally provided with chosen statistical measures. The statistical analyses were carried out for Poland's main national parks which represent the natural stands of temperate climate.
\end{abstract}

\section{Introduction}

Forests cover about $31 \%$ of the land [1], and their role in the natural environment and in human activities is essential. For example, forests have a significant influence on the composition of dust and atmospheric gases, air and soil temperature, the amount of water present, and forested areas, both in soil and in vegetation cover. Forests also play an important role in the exchange of water between the soil and the atmosphere. Forest management requires timely and accurate information on forests [2] and remote sensing methods have been used for forest inventory for decades [3-5]. A major problem in forest research is the diversity of ecosystems, which provides for the possible couplings of various physical and biological processes, especially when there is a need of mesoscale assessments. It is therefore essential how large areas can be represented by the data (satellite, terrestrial, and statistical assessment) and what time resolution measurements are performed with. For all these reasons, studies on forest ecosystem, have always required considerable effort and resources.

In remote observations of forests, spectral analysis allows for the determination of various biophysical parameters such as NDVI, LAI (leaf area index), FCover (fraction of vegetation cover), FAPAR (fraction of absorbed photosynthetically active radiation), and LAIxCab (canopy chlorophyll content of A and B types) [6-9]. These indicators and their properties are well described in the literature $[10,11]$, so they will not be detailed discussed here. Applying these vegetation indices, it is possible to determine quite accurately, both quantitatively and qualitatively, the state of vegetation on the Earth's surface. The vegetation indices are closely related to 
the fundamental energy and biological and physical phenomena, but at the same time they are distant from the direct parametric assessments. Thus, one uses vegetation indices to evaluate more complex quantities, such as biomass and its productivity, the ability to bind water with vegetation or even with soil under the trees, diverse statistical characteristics of trees (e.g., diameter at breast height, cross-sectional area), evapotranspiration, and the ability of carbon binding.

It is worth mentioning that diverse sophisticated vegetation indices as well as their processors specialized to specific satellite imagery are still developed giving new impetus to the development of better methods for classification of forests.

High resolution observations are most commonly associated with the necessity of obtaining and processing large amounts of data $[10,11]$ while (contrary to popular opinion) they are usually rare, and even incidental, limited to the time of satellite flight, depending on cloud conditions, research cost, and so forth. Therefore, it is generally accepted that the satellite data with an average or low spatial resolutions are also very valuable, because such data are easily and regularly accessible, often at no cost, as open to the public.

It should be also emphasized that high spatial resolution, in observing the Earth's surface, including the forest ecosystems, is not the only or even the most important criterion of quality satellite observations. Increasing spatial resolution capabilities have caused a sharp increase in the amount of data and resources needed to process them, resulting in increased costs of research and investment of human labour. A suitable compromise in the selection of spatial and temporal resolutions is therefore essential [12, 13]. An example of a large-scale, technologically advanced satellite program designed to study the content of water in the soil, as well as the amount of the water bounded with vegetation, including forests, is the mission SMOS (soil moisture and ocean salinity), working in the range of microwave radiation $(1.400-1.427 \mathrm{GHz})$, which ensures the relevance of the large-area assessments. At the same, time resolving power of this probe is relatively low, about 32 to $50 \mathrm{~km}$ [14]. Inventory of forest environments obviously must also involve high resolution observations, including ground-based data, but such observations are not reliable enough, if they do not enable comparisons of separate and isolated areas, such as, for instance, various national parks. Often there is not only the need for an exact assessment of detailed information as, for example, distribution of tree species, but also, especially in the climate-forest interaction studies, some essential forest features as forest type or forest state, which can sometimes be difficult to be distinguished in too small or directly neighbouring forest areas. Inexpensive and convenient observations at large scales and meso-(i.e., intermediate) scales are and will always be necessary for many regional assessments. Therefore, medium and low resolution methods of forest ecosystem observations are intensively developed together with high resolution ones.

The aim of this study was to develop an inexpensive method to distinguish between three selected classes of forest: coniferous, deciduous, and mixed by means of satellite images of low (in terms of forest applications) resolution, based on seasonal changes in selected indicators of vegetation. The
TABLE 1: Land cover class in the studied area according to the Corine Land Cover, 2006 database.

\begin{tabular}{lcc}
\hline Land cover & Area $\left[\mathrm{km}^{2}\right]$ & Percentage [\%o] \\
\hline Anthropogenised area & 10.8 & 4.83 \\
Agricultural area & 358.4 & 160.44 \\
Deciduous forests & 587.9 & 263.18 \\
Coniferous forests & 716.9 & 320.93 \\
Mixed forests & 276.8 & 123.91 \\
Forests in changes & 68.4 & 60.62 \\
Pastures & 1.6 & 0.72 \\
Wetlands & 212.2 & 95.00 \\
Water areas & 0.9 & 0.40 \\
\hline Total & 2233.8 & 1000 \\
\hline
\end{tabular}

results of this method could be used, for example, to validate the aforementioned SMOS data or used in the climate-forest interaction investigations. It was assumed that the classification results could be easily updated annually, which is much more frequent than, for example, the spatially detailed, and widely used in European Union, Corine Land Cover database [15], which has been updated at great expense every few years (namely, in 1990, 2000, and 2006). Therefore, the Corine Land Cover data should be considered as independent of seasonal changes and may serve for validating analysis with the use of lower resolutions satellite sensors as MERIS, MODIS, SPOT, CHRIS, and so forth.

In addition, the aim of this work was to motivate the interest in remote observations at medium and low resolution, which are inexpensive, relatively easily accessible, and very well equipped with free tools available for data analysis. Particular value of spectral analysis is an opportunity of evaluating evapotranspiration of forest areas, as it is one of the most important elements of water balance on forest area.

\section{Study Area}

The statistical analyses were carried out for seven national parks of Poland that represent the typical, natural stands of temperate climate, namely, the Białowieski National Park, the Biebrzański National Park, the Bolimowski National Park, the Kampinoski National Park, the Kozienicki National Park, the Roztoczański National Park, and the Świętokrzyski National Park. The location of these parks on the map of Poland is shown in Figure 1. The total area of studied parks was about $2234 \mathrm{~km}^{2}$, including about $1582 \mathrm{~km}^{2}$ of coniferous, deciduous, and mixed forests which is more than $70 \%$ of the examined area. The detailed information on land cover within the whole studied area is given in Table 1 . As a test area, the Kampinoski National Park was selected, which is located in the vicinity of Warsaw, capital of Poland. The total area of this park is about $385 \mathrm{~km}^{2}$.

\section{Satellite and Test Data Description}

The study was based on low spatial resolution data FR_1P and FR_2P of the ENVISAT/MERIS (medium resolution imaging 


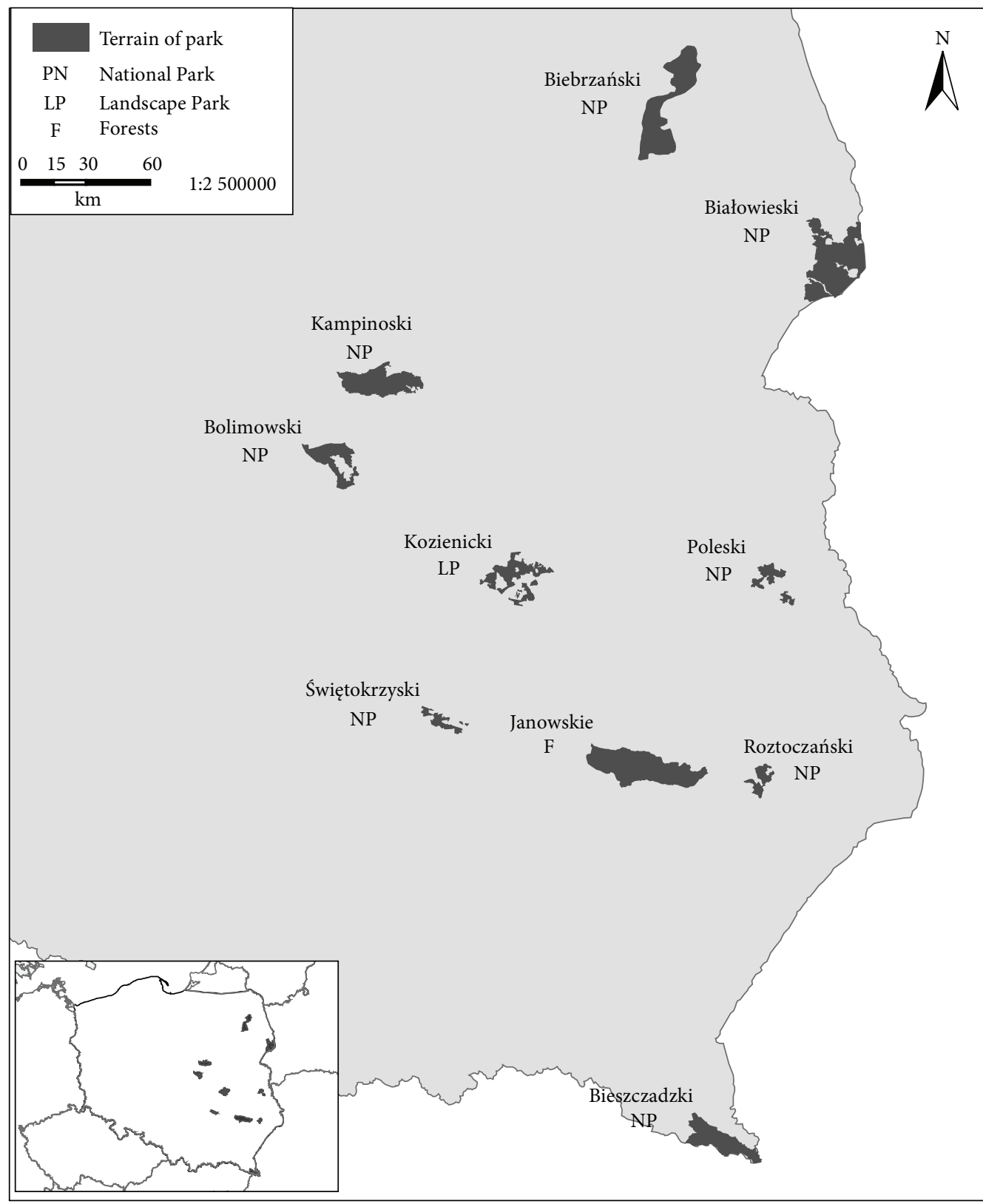

Figure 1: Location of study area on the map of Poland.

spectrometer, spatial resolution $300 \mathrm{~m}$ ) satellite sensor of levels, as well as on similar MOD15A2 MOD13A2 data of the MODIS/TERRA (moderate resolution imaging spectroradiometer, spatial resolution $1000 \mathrm{~m}$ ). The selection of images was guided by the above-described reasons, as well as the by the evaluation of image quality in terms of the instantaneous cloud cover. MODIS images were obtained free of charge directly from the WIST site (The Warehouse Inventory Search Tool). MERIS images were also obtained free of charge in cooperation with the Space Research Centre, Polish Academy of Sciences, the Cat-1 in the frame of project AO-3275. All the preliminary operations and the analysis of vegetation indices from MERIS images were performed using the Visat (BEAM) program from Brockmann Consult and Contributors, shared free of charge by the European Space Agency (ESA). To prepare remote images of good quality before the main analysis, it was necessary to perform carefully many timeconsuming operations such as calibration, scaling, and image orthorectification. Detailed description of these necessary, but time-consuming analyses, is not possible in this paper.

Figures 2 and 3 show examples of spatial distributions of exemplary indices (FAPAR_MERIS and FAPAR_MODIS) in the area covering the whole of the satellite image.

When performing the spatial analysis, the GIS (Geographical Information Systems) tools were also used, namely ArcGIS 9.1, while the statistical calculations were made in the Statistica package. The Corine Land Cover, 2006 database, was used to take into account only natural stands from the 


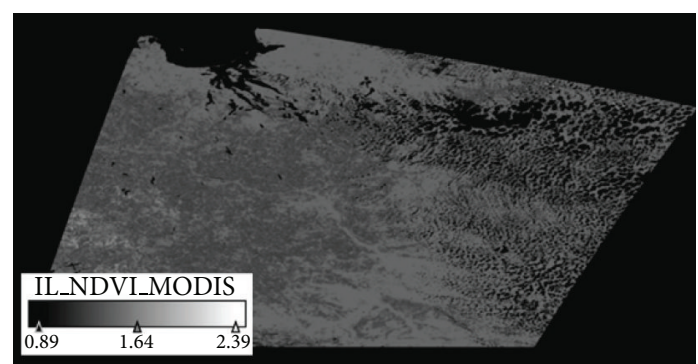

FIGURE 2: Example of spatial distribution of the IL_NDVI_MODIS vegetation index obtained from the MERIS image, on July 3, 2008, and on April 2, 2009.

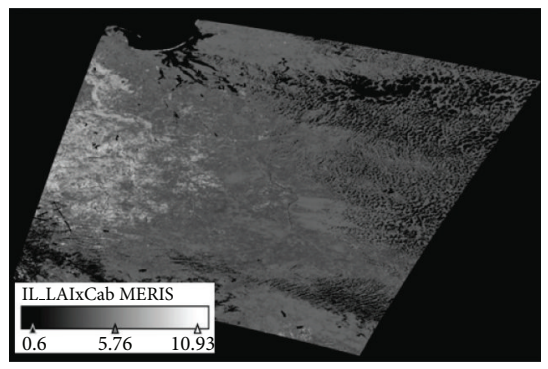

FIGURE 3: Example of spatial distribution of the IL_LAIxCab_MERIS vegetation index obtained from the MODIS image, on July 32008 , and on April 22009.

above-described seven national parks. To assure the best quality of results of GIS and statistical analyses, all pixels taken for calculations were carefully filtered. The area, which included the above-mentioned three types of forests and was not obscured by clouds in satellite images, had an area of approximately $875 \mathrm{~km}^{2}$.

\section{Results and Discussion}

The demonstration of significant differences between the three forest types coniferous, deciduous, and mixed in remote sensing data of relatively low resolution satellite images required proper selection of vegetation indices. This was made by carefully analyzing the major seasonal changes of vegetation indices, ranging from classical vegetation indeces NDVI and LAI, to much more sophisticated, such as FAPAR, fCover, LAIxCab, and MGVI. After preliminary analysis described in the previous section, seven different vegetation indices were chosen for the study: NDVI_MERIS, FAPAR_MERIS, LAI_MERIS, LAIxCab_MERIS, NDVI_MODIS, and IL_LAI_MODIS.

In view of the fact that most conifer trees do not lose their needles, one can expect that their vegetation indices show much smaller seasonal changes than those deciduous ones $[16,17]$.

As an example of such behaviour, histograms presented in Figures 4 and 5 show the typical distributions of the FAPAR_MERIS index values in April 2, 2009 and July 3, 2009 over area of the Kampinoski National Park. As expected, the temporal variability of this index in the case of deciduous

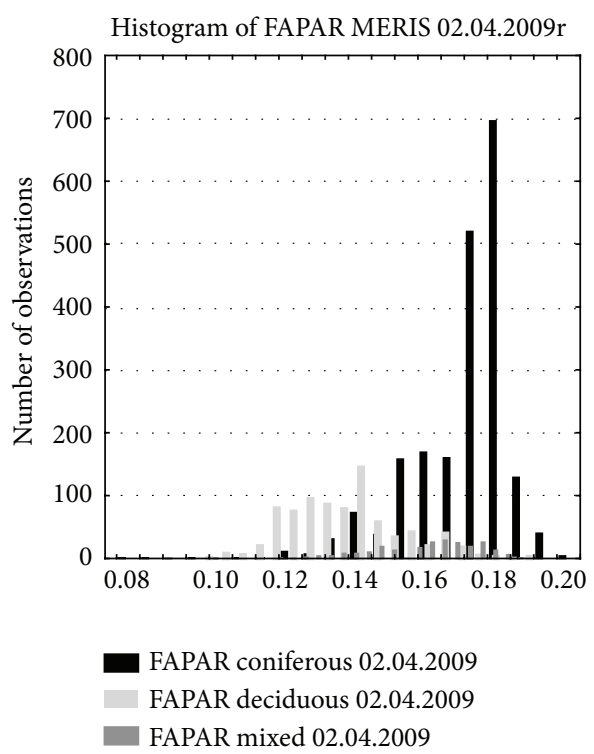

FIgURE 4: Multiple histogram of the FAPAR MERIS vegetation index for the photograph on April 2, 2009.

forests is much higher than that in case of coniferous one. In addition, one can notice that the value of the FAPAR index in April for coniferous forests is greater than that for deciduous forests, while in July the FAPAR index value is greater for deciduous forests. As it can be seen in Figures 4 and 5, indices for mixed forests take, in both cases, intermediate values as compared with those for deciduous and coniferous ones.

This justifies the use of some indices based on seasonal changes in vegetation for the classification of forest types. In this work, the ratios of particular vegetation indices determined in July and April, which are the middle and the beginning of a vegetation season in Poland, were calculated. These ratios of vegetation indices are further known briefly as ratio indices, and indicated by the letters IL. For example,

$$
\begin{aligned}
& \text { IL_FAPAR_MERIS } \\
& =\frac{\text { value of FAPAR from MERIS in July }}{\text { value of FAPAR from MERIS in April }} .
\end{aligned}
$$

It is worth noting that in order to perform such calculations it was necessary to ensure that pixels values determined in July and April were taken exactly from the same place.

The similar histograms to those for FAPAR were obtained also for all seven above-listed vegetation indices calculated from MODIS and MERIS satellite images. For all analysed national parks, the same marked tendency has been observed: ratio indices determined for coniferous forests had the smallest mean values, those determined for deciduous forests were the largest, whereas those obtained for mixed forests were intermediate. The average values of ratio indices calculated from the whole area of national parks are presented in Figure 6, whereas Table 2 shows the mean values of ratio indices calculated both for the whole area and separately for each national park. 

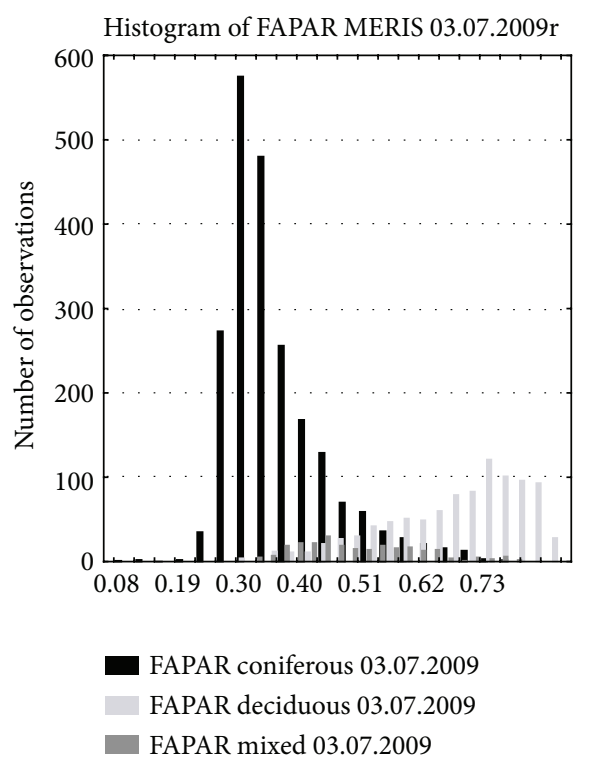

FIGURE 5: Multiple histogram of the FAPAR MERIS vegetation index for the photograph on July 3, 2009.
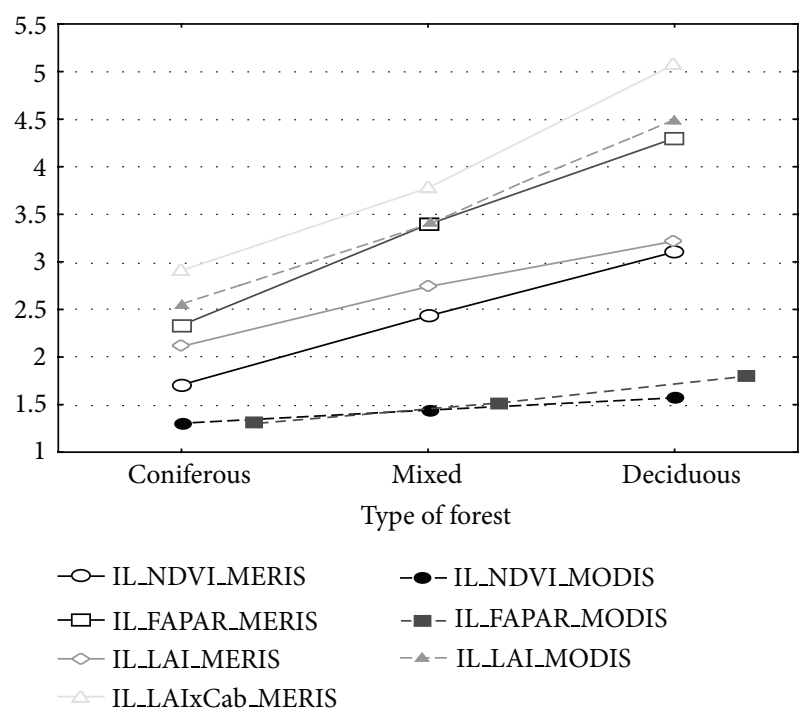

FIGURE 6: The average values of ratio indices calculated from the whole area of national parks under study.

The distinct differences in mean values of ratio indices of specific forest type made it possible to use the ratio indices for classification purposes. To do so, also the ranges of all ratio indices corresponding to different forest types were determined for the whole studied area and separately for each national park. Determination of precise limits of the ranges of all ratio indices for the selected type of forest was a difficult task because the ranges of these indices overlap each other due to natural variations in the pixel values. One could expect that the ranges of ratio indices obtained from the whole study area were more reliable; therefore they were used to obtain the spatial distributions of the forest type in the test area.
TABLE 2: The means of ratio indices for the studied types of forests in Poland.

\begin{tabular}{|c|c|c|c|}
\hline \multirow{2}{*}{ Ratio index } & \multicolumn{3}{|c|}{ Forest type } \\
\hline & Coniferous & Mixed & Deciduous \\
\hline IL_NDVI_MERIS & 1.74 & 2.51 & 3.32 \\
\hline IL_FAPAR_MERIS & 2.43 & 3.48 & 4.38 \\
\hline IL_LAI_MERIS & 2.16 & 2.72 & 3.29 \\
\hline IL_LAIxCab_MERIS & 2.88 & 3.85 & 5.21 \\
\hline IL_NDVI_MODIS & 1.31 & 1.45 & 1.57 \\
\hline IL_FAPAR_MODIS & 1.28 & 1.46 & 1.74 \\
\hline IL_LAI_MODIS & 2.43 & 3.14 & 4.14 \\
\hline PIXEL NUMBER & 7201 & 2385 & 3860 \\
\hline
\end{tabular}

Table 3 presents the limits of ranges of ratio indices with the corresponding classification accuracy of the mixed forests.

Ranges of ratio indices when applied to the classification of forest type in the Kampinoski National Park led to surprisingly good results both for coniferous and deciduous forests. The obtained accuracy ranged from about $60 \%$ to about $90 \%$, depending on chosen ratio index, which could be consider as good result $[18,19]$.

Tables 4 and 5 show the results of the classification for different types of forest for the test site, that is, the Kampinoski National Park using ratio indices calculated on the basis of MODIS and MERIS images, respectively.

The results presented in Tables 3 and 4 indicate a reasonably good classification accuracy of deciduous and coniferous forests for both types of images and apparently lower classification accuracy of mixed forests. The last effect can be attributed to too coarse spatial resolution of remote imagery. It is worth noting that the classification accuracy of the mixed forests was significantly improved when MERIS images with better spatial resolution (more than three times) were used. It should also be stressed that the highest classification accuracy of the mixed forests was obtained using IL_FAPAR_MODIS and IL_FAPAR_MERIS indices based on the FAPAR vegetation index. In order to check the proposed method spatially, also the maps at test area were drawn showing spatial distribution of the forest types obtained by means of the low resolution imagery and then compared with true maps of forests from the Corine Land Cover database. As can be seen in Figure 7, surprisingly good fit of both kinds of forest type distributions was obtained, in particular in areas with large surface and regular shape. It can be seen again that the distributions of forest types based on vegetation indices derived from satellite imagery of MERIS sensor show a much better fit than those of the MODIS sensor.

\section{Summary and Conclusions}

This paper describes a rapid and inexpensive method of the annual classification of forest ecosystems into three categories: coniferous forests, mixed forests, and deciduous ones, using optical satellite images of MODIS/TERRA and MERIS/ENVISAT sensors with low spatial resolution. Direct use of vegetation indices such as NDVI, FAPAR, and LAI 

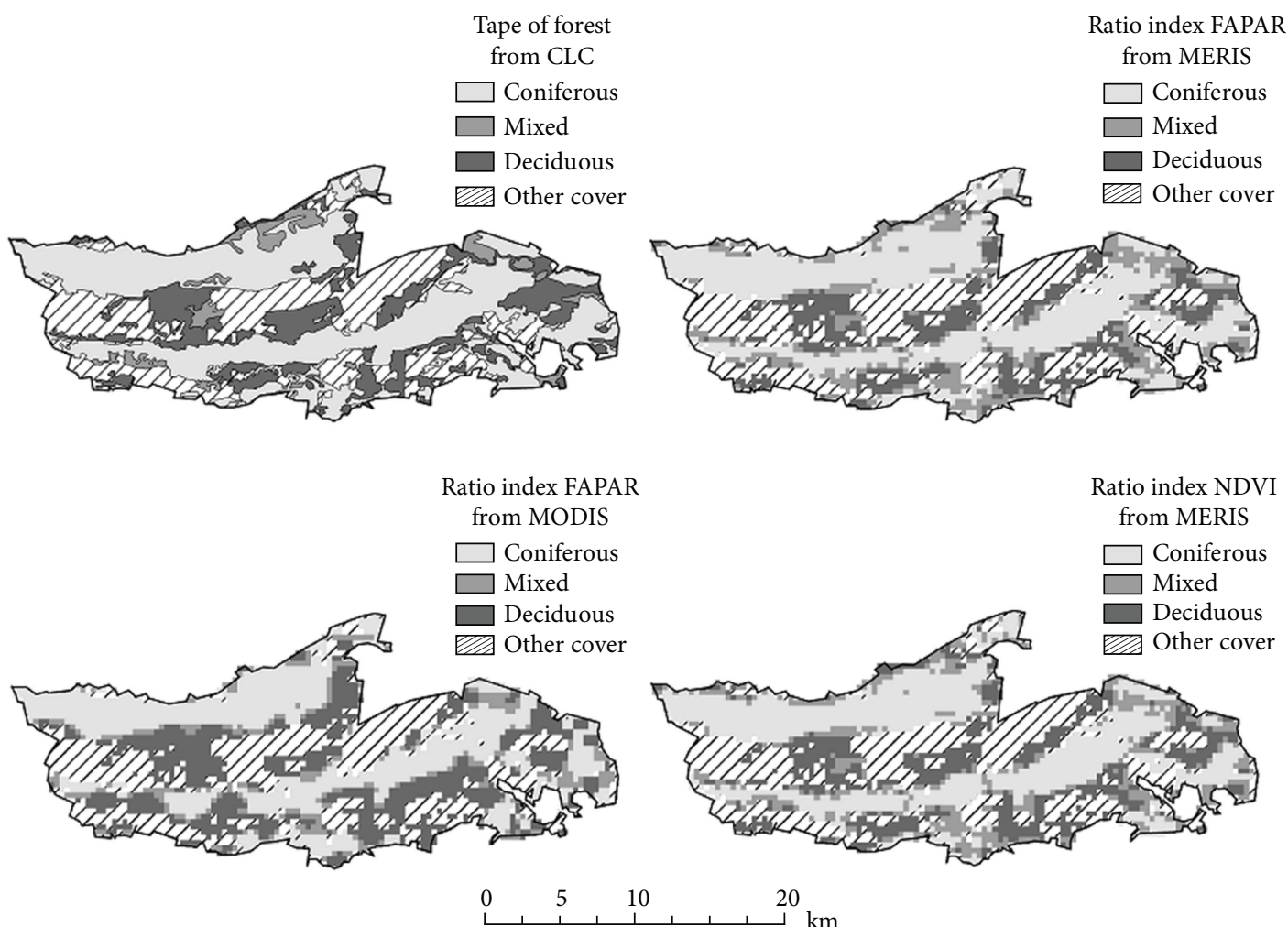

FIGURE 7: Comparison of the classification results of forest types obtained using ratio indices with the spatial distribution obtained on the basis of the accurate data from CLC database. Example based on FAPAR NDVI vegetation indices calculated from MERIS and MODIS images, with resolutions $300 \mathrm{~m}$ and $1000 \mathrm{~m}$, respectively, in the area of the Kampinoski National Park.

TABle 3: Limits indices for the studied types of forest and the classification accuracy for the mixed forests in Poland.

\begin{tabular}{lcccc}
\hline Ratio index & & Forest type & Deciduous & Mixed forest classification accuracy \\
\hline IL_NDVI_MERIS & Coniferous & Mixed & $>2.60$ & 50.2 \\
IL_FAPAR_MERIS & $<1.75$ & $1.75-2.60$ & $>3.75$ & 51.9 \\
IL_LAI_MERIS & $<2.35$ & $2.35-3.75$ & $>2.65$ & 32.8 \\
IL_LAIxCab_MERIS & $<2.05$ & $2.05-2.65$ & $>3.80$ & 45.6 \\
IL_NDVI_MODIS & $<2.90$ & $2.90-3.80$ & $>1.48$ & 30.9 \\
IL_FAPAR_MODIS & $<1.33$ & $1.33-1.48$ & $>1.55$ & 18.6 \\
IL_LAI_MODIS & $<1.38$ & $1.38-1.55$ & $>3.25$ & 17.2 \\
\hline
\end{tabular}

for this purpose did not provide sufficient accuracy, even in the case of high-resolution satellite observations. In this study, it was decided to use seasonal changes in these indices. The use of ratios of vegetation indices (the ratio indices) calculated from satellite observations performed in July and April was here proposed. The typical ranges of values of selected ratio indices for coniferous, deciduous, and mixed forests in natural stands were determined. The results of analysis performed in the work show that limits of ranges of ratio indices developed in this work may be used for efficient forest classification using low resolution satellite imagery. The method was verified at the Kampinoski National Park area using the Corine Land Cover database. Good accuracy of classification was obtained despite the relatively low spatial resolutions used in the analysis comparing to those used commonly in the satellite observations of forest ecosystems. Lack of full agreement of the classification results with the true spatial distribution of forests may also be due to the fact that boundaries of different types of forest are not sharp but blurred. The transition between forest specified in the Corine Land Cover database as coniferous and mixed in nature takes place smoothly. Moreover, in areas where there is a large variation of the forest type, the corresponding pixel in satellite image may be located on the border between two or even three forests and thus incorrectly classified. The highest classification accuracy of the mixed forests was obtained using ratio indices based on FAPAR. 
TABLE 4: The results of the classification of studied types of forest in the Kampinoski National Park using ratio indices calculated on the basis of MODIS images.

\begin{tabular}{lccccccccc}
\hline Ratio index & \multicolumn{3}{c}{ IL_NDVI_MODIS } & \multicolumn{3}{c}{ IL_FAPAR_MODIS } & \multicolumn{3}{c}{ IL_LAI_MODIS } \\
Forest type & Coniferous & Mixed & Deciduous & Coniferous & Mixed & Deciduous & Coniferous & Mixed & Deciduous \\
\hline Classification accuracy [\%] & 63.6 & 10.4 & 62.5 & 61.8 & 15.6 & 67.8 & 61.8 & 14.4 & 66.4 \\
\hline
\end{tabular}

TABLE 5: The results of the classification of studied types of forest in the Kampinoski National Park using ratio indices calculated on the basis of MERIS images.

\begin{tabular}{|c|c|c|c|c|c|c|}
\hline Ratio index & \multicolumn{3}{|c|}{ IL_NDVI_MERIS } & \multicolumn{3}{|c|}{ IL_FAPAR_MERIS } \\
\hline Forest type & Coniferous & Mixed & Deciduous & Coniferous & Mixed & Deciduous \\
\hline Classification accuracy [\%] & 75.1 & 52.4 & 77.3 & 77.7 & 60.0 & 72.8 \\
\hline Ratio index & \multicolumn{3}{|c|}{ IL_LAI_MERIS } & \multicolumn{3}{|c|}{ IL_LAIxCab_MERIS } \\
\hline Forest type & Coniferous & Mixed & Deciduous & Coniferous & Mixed & Deciduous \\
\hline Classification accuracy [\%] & 76.7 & 22.0 & 71.3 & 68.7 & 36.4 & 90.0 \\
\hline
\end{tabular}

In view of further global-scale research of the hydrological conditions and evapotranspiration at forest areas, the use of MODIS optical data and very low resolution microwave SMOS data is considered. The results obtained in this work could be applied in such studies.

\section{Acknowledgments}

The authors acknowledge the support received under a grant financed by the European Union under the European Social Fund, which is awarded by the Centre for Advanced Studies, Warsaw University of Technology in the framework of the project "Warsaw University of Technology Development Program."

\section{References}

[1] Food and Agriculture Organization of the United Nations, http://www.fao.org/news/story/en/item/40893/icode/ .

[2] M. C. Hansen, S. V. Stehman, P. V. Potapov et al., "Humid tropical forest clearing from 2000 to 2005 quantified by using multitemporal and multiresolution remotely sensed data," Proceedings of the National Academy of Sciences of the United States of America, vol. 105, no. 27, pp. 9439-9444, 2008.

[3] M. C. Hansen, S. V. Stehman, and P. V. Potapov, "Quantification of global gross forest cover loss," Proceedings of the National Academy of Sciences of the United States of America, vol. 107, no. 19, pp. 8650-8655, 2010.

[4] M. C. Hansen, S. V. Stehman, and P. V. Potapov, "Reply to Wernick et al.: Global scale quantification of forest change," Proceedings of the National Academy of Sciences of the United States of America, vol. 107, no. 38, p. E148, 2010.

[5] J. Zawadzki, C. J. Cieszewski, M. Zasada, and R. C. Lowe, "Applying geostatistics for investigations of forest ecosystems using remote sensing imagery," Silva Fennica Monographs, vol. 39, no. 4, pp. 599-617, 2005.

[6] F. Baret, K. Pavageau, Bacour et al., "Algorithm Theoretical Basis Document for MERIS Top of Atmosphere Land Prod. (TOA-VEG)," INRA-Novelties, 2009, http://www.brockmannconsult.de/beam/plugins.html.
[7] C. Bacour, F. Baret, D. Béal, M. Weiss, and K. Pavageau, "Neural network estimation of LAI, fAPAR, fCover and LAI $\times$ Cab, from top of canopy MERIS reflectance data: principles and validation," Remote Sensing of Environment, vol. 105, no. 4, pp. 313-325, 2006.

[8] N. Gobron, B. Pinty, F. Mélin et al., "Evaluation of the MERIS/ENVISAT FAPAR product," Advances in Space Research, vol. 39, no. 1, pp. 105-115, 2007.

[9] R. B. Myneni, S. Hoffman, Y. Knyazikhin et al., "Global products of vegetation leaf area and fraction absorbed PAR from year one of MODIS data," Remote Sensing of Environment, vol. 83, no. 1-2, pp. 214-231, 2002.

[10] J. W. Rouse, R. H. Haas, J. A. Schell, and D. W. Deering, "Monitoring vegetation systems in the great plains with ERTS," in Proceedings of the 3rd ERTS Symposium. NASA SP-351, pp. 309-317, NASA, Washington, DC, USA, 1973.

[11] R. Nemani, L. Pierce, S. Running, and L. Band, "Forest ecosystem processes at the watershed scale: sensitivity to remotelysensed leaf area index estimates," International Journal of Remote Sensing, vol. 14, no. 13, pp. 2519-2534, 1993.

[12] A. H. Strahler, C. E. Woodcock, and J. A. Smith, "On the nature of models in remote sensing," Remote Sensing of Environment, vol. 20, no. 2, pp. 121-139, 1986.

[13] P. Treitz and P. Howarth, "High spatial resolution remote sensing data for forest ecosystem classification: an examination of spatial scale," Remote Sensing of Environment, vol. 72, no. 3, pp. 268-289, 2000.

[14] ESA-SMOS Earth Explorers, http://www.esa.int/esaLP/ ESAS7C2VMOC_LPsmos_0.html.

[15] M. Bossard, J. Feranec, and J. Otahel, "Corine land cover technical guide-Addendum," Technical Report 40, EEA, Copenhagen, Denmark, 2000.

[16] A. M. Jönsson, L. Eklundh, M. Hellström, L. Bärring, and P. Jönsson, "Annual changes in MODIS vegetation indices of Swedish coniferous forests in relation to snow dynamics and tree phenology," Remote Sensing of Environment, vol. 114, no. 11, pp. 2719-2730, 2010.

[17] Q. Zhang, X. Xiao, B. Braswell et al., "Characterization of seasonal variation of forest canopy in a temperate deciduous broadleaf forest, using daily MODIS data," Remote Sensing of Environment, vol. 105, no. 3, pp. 189-203, 2006. 
[18] S. Lewiński, "Object based classification of middle resolution MODIS satellite image, first results," Archiwum Fotogrametrii, Kartografii i Teledetekcji, vol. 21, pp. 211-219, 2010.

[19] S. Lewiński, "Applying fused multispectral and panchromatic data of Landsat ETM+ to object oriented classification," in New Developments and Challenges in Remote Sensing, Millpress, Rotterdam, The Netherlands, 2007. 

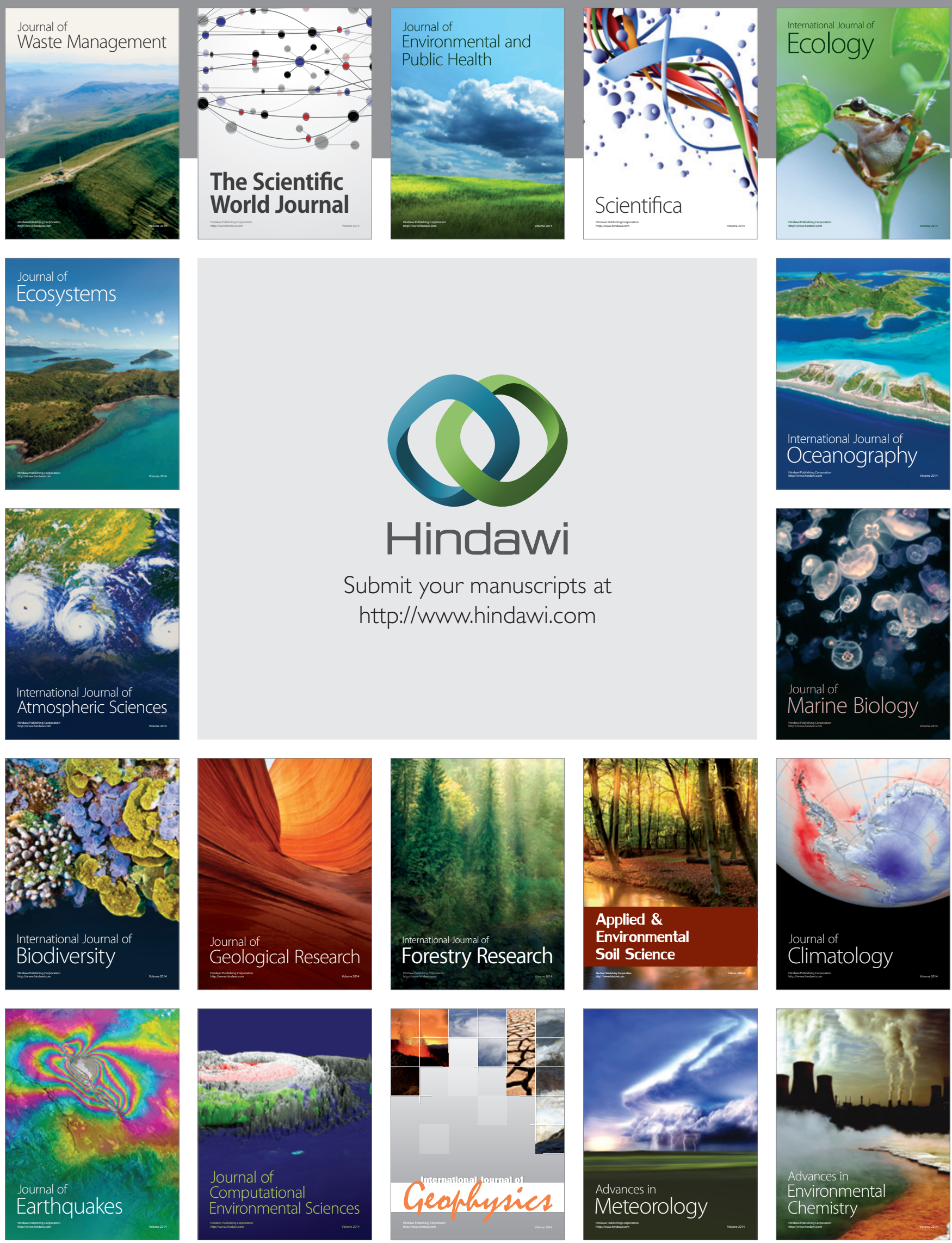